Results 656 shoulder workloads were analysed. At followingend, following-span median was 12.5 years, age median was 42 years, $60 \%$ were women, $85 \%$ had non-university academic level and $77 \%$ had non-administrative positions. Age, handedness, academic level, work type and mood disorders were proved as significant or as confounding covariates within the final model. $4 \times 10^{3}$ cumulative-effective working hours of shoulder repetitiveness exposure was established clearly as threshold (adjusted HR=1.93; 95\% CI: 1.04 to 3.59).

Discussion Taking real action in developing world should be addressed towards effective primary prevention, which means that no worker should be exposed more than repetitiveness threshold, in order to eliminate shoulder's CTD. On the other hand, proved threshold overpassing shall confirm workrelated causation in injured workers within compensation processes.

\section{ASSESSMENT OF HUMAN EXPOSURE TO 1- NITROPYRENE BY MEANS OF THE DETERMINATION OF HYDROXYNITROPYRENES IN 896 URINE SAMPLES}

${ }^{1} \mathrm{G}$ Tranfo*, ${ }^{1} \mathrm{D}$ Pigini, ${ }^{1} \mathrm{E}$ Paci, ${ }^{2} \mathrm{~L}$ Bauleo, ${ }^{2} \mathrm{C}$ Ancona, ${ }^{2} \mathrm{~F}$ Forastiere. ${ }^{1}$ INAIL Research, Department of Occupational Medicine, Epidemiology and Hygiene, Research Area of Monte Porzio Catone, Rome, Italy; ${ }^{2}$ Lazio Regional Health Service, Department of Epidemiology, Rome, Italy

\subsection{6/oemed-2018-ICOHabstracts.340}

Introduction Polycyclic aromatic hydrocarbons (PAHs) are ubiquitous organic pollutants, whose sources include traffic emissions derived from diesel/gasoline vehicles. Monohydroxylated PAHs (OH-PAHs), urinary metabolites of PAHs, were used as biomarkers of PAHs exposure. 1-Nitropyrene is a molecular marker for diesel exhaust, a significant contributor to the toxicity associated with particulate matter. Urinary metabolites of 1-Nitropyrene were recently evaluated for their utility as markers of exposure to diesel exhaust.

Methods Among the three isomers 3-, 6-, and 8-hydroxy-nitropyrene (3-, 6-, 8-OHNPy), 6-OHNPy was selected for this study as it is the most abundant found in human urine. 896 urine samples were collected from subjects randomly selected from municipality registers of Civitavecchia (Central Italy) as a part of the 'ABC Human Biomonitoring study' and tested after enzymatic hydrolysis of the glucuronic acid conjugates using HPLC-MS/MS. Pure standard of 6-OHNPy was purchased following custom synthesis and deuterium labelled 1-hydroxypyrene was used as internal standard for quantitative determination.

Result Results show that more than 50\% of the 896 samples did not contained detectable concentrations of total hydroxy-nitropyrenes measured as 6-OHNPy $(<0.0005 \mu \mathrm{g} / \mathrm{g}$ of creatinine), while 383 samples showed measurable levels, in the range 3.81$0.0005 \mu \mathrm{g} / \mathrm{g}$ of creatinine. Mean value is $0.091 \mu \mathrm{g} / \mathrm{g}$ of creatinine. Discussion This is the first large study reporting urinary levels of 6-hydroxynitropyrene in subjects non-occupationally exposed to 1-nitro-pyrene. Previous studies showed that this biomarker is scarcely influenced by smoking. As the performances of the method allow environmental exposure assessment, where high sensitivity is needed, it can be used with even greater confidence for studies of occupational exposure assessment to diesel exhaust, where higher exposure levels are expected: the urinary levels here reported can be considered as reference values to be compared to the levels produced by occupational exposures.
111 THE IMPACT OF INTRODUCING NATIONAL POLICIES OF IMPROVING WORKING CONDITIONS ON OVERWORKRELATED CARDIOVASCULAR MORTALITY IN TAIWAN

Ro-Ting Lin*. College of Public Health, China Medical University, Taichung, Taiwan

10.1136/oemed-2018-ICOHabstracts.341

Introduction Cardiovascular diseases (CVDs) are the leading cause of death globally.

Occupational related risk factors for CVD incidence and mortality include long working hours, high job stress, and low employment opportunities. 'Overwork' or 'Karoshi' has since been a major occupational health concern for Asian workers. In response to public's worries of long working hours and low salary in Taiwan, Taiwanese government vigorously amended standard working hours, holiday, and basic salary under the Labour Standard Act and the criteria to recognise overwork-related CVDs during 2015-2016. We investigated the ecological association among national policies, working conditions, and overwork-related CVDs.

Methods We collected data on the working conditions and overwork-related CVDs for each industry sector and identify the high-risk group. We estimated the combined effect of national policies and working conditions on overwork-related CVDs for each industry sector and further estimated the expected number of overwork-related CVDs to investigate the impact of introducing the new policies. We finally measured the extent of preventable CVDs for each industry sector as a result of introducing new policies and improving working conditions.

Result Our analysis covered 11.27 million workers from 17 industry sectors in Taiwan. We found consistent and plausible correlations between the implementation of new policies, the working conditions, and the number of recognised overworkrelated CVDs. Compared to workers in the education sector, employees in the support service and manufacturing sectors had greater likelihoods of overwork-related CVDs. Such gap was particularly obvious in their monthly working hours by a difference of 50 hours per month.

Discussion In view of Taiwan's working environment, reducing working hours is the key element to reducing the burden of overwork-related CVDs. Our result can contribute to the government's decision-making process and society's understanding of preventable overwork-related CVDs in each industry sector by providing justification for proceeding to new policies.

\section{COMBINING LEAD EXPOSURE MEASUREMENTS AND EXPERTS' OPINION THROUGH A BAYESIAN FRAMEWORK}

${ }^{1}$ Dong-Hee Koh*, ${ }^{2}$ Sang-Gil Lee, ${ }^{3}$ Hwan-Cheol Kim, ${ }^{4}$ Ju-Hyun Park, ${ }^{5}$ Sangjun Choi, ${ }^{1}$ Hyejung Jung, ${ }^{6}$ Dong-Uk Park. ${ }^{1}$ Department of Occupational and Environmental Medicine, International St. Mary's Hospital, Catholic Kwandong University, Incheon, Korea; ${ }^{2}$ Occupational Safety and Health Research Institute, Korea Occupational Safety and Health Agency, Ulsan, Korea; ${ }^{3}$ Department of Occupational and Environmental Medicine, Inha University, Incheon, Korea; ${ }^{4}$ Department of Statistics, Dongguk University, Seoul, Korea; ${ }^{5}$ Department of Occupational Health, Catholic University of Daegu, Daegu, Korea; ${ }^{6}$ Department of Environmental Health, Korea National Open University, Seoul, Korea

\subsection{6/oemed-2018-ICOHabstracts.342}

Objectives CAREX (CARcinogen EXposure) is a carcinogen surveillance system employed in many countries. To initiate 
Korean CAREX, we focused on estimating the exposure intensity of lead across industries, which is a suspected carcinogen. Methods We extracted airborne lead measurements from the work environment measurement database (WEMD) which is the Korean nationwide measurement database. In addition, we elicited the experts' opinion about lead exposure intensity across industries by conducting a questionnaire. Experts provided estimates of lead exposure levels as the boundary of the 5 th and 95th percentiles. We assumed that experts provided their estimates based on the assumption of log-normal distributions of exposure. First, for each industry, estimates of logtransformed geometric means (logGM) and log-transformed geometric standard deviations (logGSD) were extracted from the experts' responses, followed by combining them to quantify the experts' prior Normal-Inverse-Gamma prior distribution. Then, the corresponding $\log G M$ and $\log$ GSD from lead measurement data for each industry were updated with the experts' prior distribution through a Bayesian framework, yielding posterior distributions of $\log \mathrm{GM}$ and $\log \mathrm{GSD}$.

Results WEMD contains 83035 airborne lead measurements collected between 2002-2007. Total 17 occupational hygiene professionals with more than 20 year experience provided lead exposure estimates. In industries where measurement data is abundant, the measurement data dominate the posterior exposure estimates, while in industries with a limited number of measurements, experts' opinion played an important role in determining posterior exposure estimates. For example, rubber manufacturing industry with 246 measurements (GM 1.72; GSD 1.94) and 6 experts' responses (GM 0.79; GSD 6.73) showed posterior exposure estimates of GM 1.60 and GSD 2.34 .

Conclusions Our method of estimating the exposure intensity of CAREX may introduce an unbiased approach to the development process by utilising both prior knowledge of experts and measurement data simultaneously. In addition, it will supply a framework for future updates.

\section{ESTIMATION OF LEAD EXPOSURE PREVALENCE IN KOREAN POPULATION THROUGH COMBINING MULTIPLE EXPERTS' JUDGMENT BASED ON OBJECTIVE DATA SOURCES}

${ }^{1}$ Dong-Hee Koh*, ${ }^{2}$ Sang-Gil Lee, ${ }^{3}$ Hwan-Cheol Kim, ${ }^{4}$ Ju-Hyun Park, ${ }^{5}$ Sangiun Choi, ${ }^{1}$ Hyejung Jung, ${ }^{6}$ Dong-Uk Park. 'Department of Occupational and Environmental Medicine, International St. Mary's Hospital, Catholic Kwandong University, Incheon, Korea; ${ }^{2}$ Occupational Safety and Health Research Institute, Korea Occupational Safety and Health Agency, Ulsan, Korea; ${ }^{3}$ Department of Occupational and Environmental Medicine, Inha University, Incheon, Korea; ${ }^{4}$ Department of Statistics, Dongguk University, Seoul, Korea; ${ }^{5}$ Department of Occupational Health, Catholic University of Daegu, Daegu, Korea; ${ }^{6}$ Department of Environmental Health, Korea National Open University, Seoul, Korea

\subsection{6/oemed-2018-ICOHabstracts.343}

Objectives Estimation of exposure prevalences of carcinogens is important for preventing occupational cancers. For develop-

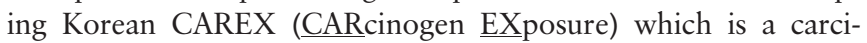
nogen surveillance system employed in many countries, as an exemplary carcinogen, we estimated lead exposure prevalences in Korean working population.

Methods We used three nationwide data sources to retrieve objective default estimates of lead exposure prevalence across industries: the work-environment monitoring database (WEMD), the special health examination database (SHED), and the Work Environment Condition Survey (WECS).
Furthermore, we surveyed experts for their judgment about lead exposure prevalence across industries after reviewing the default estimates computed from aforementioned three exposure databases. We developed various estimation methods combining experts' judgment, and then compared with each other. The 2010 Census was used as the reference population to estimate the number of lead-exposed workers for the 228 industries through multiplying the exposure prevalence with the number of workers in each industry.

Results Default estimates of exposure prevalence for 228 industries were calculated using the WEMD and SHED collected between 2009-2011, and the 2009 WECS. A total of 52 experts who have 20 or more experience in industrial hygiene practice participated in the study and provided their judgment on the lead exposure prevalences as a number for the 228 industries. Among various estimation methods, the median values of experts' responses were selected for our estimates of lead exposure prevalence in each industry. As a result, a total of 129,247 Korean workers was estimated to be exposed to lead in the year of 2010.

Conclusions We developed a method estimating exposure prevalences combining experts' judgment based on objective databases. Our effort for estimating the exposure prevalences of CAREX may introduce an unbiased approach to the development process accounting for uncertainty of exposure.

\section{BIG DATA AND OCCUPATIONAL HEALTH SURVEILLANCE: USE OF FRENCH MEDICO- ADMINISTRATIVE DATABASES FOR HYPOTHESIS GENERATION REGARDING OCCUPATIONAL RISKS IN AGRICULTURE}

${ }^{1,2}{ }^{2}$ Maugard*, ${ }^{1} D$ Bosson-Rieutort, ${ }^{2} \mathrm{O}$ François, ${ }^{1,3 \mathrm{~V}}$ Bonneterre. ${ }^{1}$ EPSP Team, TIMC-IMAG Laboratory, Grenoble-Alpes University, Grenoble, France; ${ }^{2} B C M$ Team, TIMC-IMAG Laboratory, Grenoble-Alpes University, Grenoble, France; ${ }^{3}$ Occupational Medicine and Health Dpt., CHU Grenoble-Alpes, Grenoble, France

\subsection{6/oemed-2018-ICOHabstracts.344}

Introduction Surveillance of diseases and associated exposures is a major issue in occupational health, especially for identifying new work-related diseases. In addition to classical epidemiology (hypothesis-driven studies), complementary methods relying on data mining of health insurance data must be developed for early detection of work-related diseases, without prior hypothesis.

Methods Data from the insurance fund of French agricultural workers ('Mutualité Sociale Agricole', MSA), which covers about 3 million individuals, were considered. The study population included all self-employed or employee affiliates from the 2006-2015 period. MSA holds medico-administrative databases, which include information on occupational activities as well as long-term diseases identified with ICD-10 codes. Following authorisation of MSA and of the French National Commission on Informatics and Liberty, these databases were cross-linked for the first time. After preliminary data treatments, generalised linear models and latent factor models were applied to detect over-represented statistical associations between occupational activity and long-term disease. Results were represented as p-value plots in order to highlight the key statistical signals.

Results The population covered by this study accounted for more than 2 million individuals $(n=2,250,177)$ with a majority of men $(64 \%)$ and an average age of 46 years. Within this 